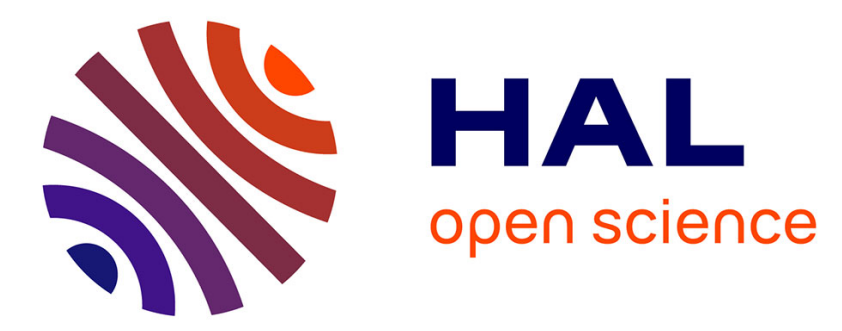

\title{
What is a just society? The answer according to the Socialistes Fraternitaires Louis Blanc, Constantin Pecqueur, and François Vidal
}

\author{
Ludovic Frobert
}

\section{- To cite this version:}

Ludovic Frobert. What is a just society? The answer according to the Socialistes Fraternitaires Louis Blanc, Constantin Pecqueur, and François Vidal. History of Political Economy, 2014, 46 (2), pp.281-306. 10.1215/00182702-2647513 . halshs-00992599

\section{HAL Id: halshs-00992599 \\ https://shs.hal.science/halshs-00992599}

Submitted on 23 Jun 2017

HAL is a multi-disciplinary open access archive for the deposit and dissemination of scientific research documents, whether they are published or not. The documents may come from teaching and research institutions in France or abroad, or from public or private research centers.
L'archive ouverte pluridisciplinaire HAL, est destinée au dépôt et à la diffusion de documents scientifiques de niveau recherche, publiés ou non, émanant des établissements d'enseignement et de recherche français ou étrangers, des laboratoires publics ou privés. 


\title{
What is a just society? \\ The answer according to the Socialistes Fraternitaires Louis Blanc, Constantin \\ Pecqueur, and François Vidal.
}

\author{
QU'EST GE QU'UNE SOCIETE JUSTE? \\ La réponse des Socialistes Fraternitaires autour de 1848 \\ (Louis Blanc, Constantin Pecqueur, François Vidal) \\ Ludovic Frobert \\ CNRS/TRIANGLE
}

Louis Blanc est sans doute l'un des plus importants représentants de la génération des socialistes français qui occupèrent le devant de la scène politique et économique au tournant de 1848. Ses articles et essais, notamment Organisation du travail, furent au cœur des polémiques passionnées du tournant des années 1840 et lors du printemps 1848, enfant terrible du gouvernement provisoire de la République, il fut le principal instigateur, avec ses collègues Constantin Pecqueur et François Vidal qu'il avait appelé à ses côtés, de l'expérience pionnière de la Commission du Luxembourg. Là, durant quelques semaines cruciales, ces trois socialistes s'employèrent à jeter les bases d'une nouvelle conception, plus fraternelle, des rapports sociaux.

Cet article tentera de démontrer que ce trio de Socialistes fraternitaires eu pour intention prioritaire d'apporter un ensemble de réponses cohérentes, tant théoriques que pratiques, à la question : qu'est ce qu'une société juste? Confrontés aux maux de la société de leur temps, et face aux lacunes des discours économiques tant libéraux que protectionnistes, ils estimaient indispensables d'agir ${ }^{1}$ en formulant un modèle complet de justice sociale, présentant des énoncés prescriptifs, normatifs et évaluatifs sur les principes et les institutions (mais aussi les comportements) d'une société juste. Portés au pouvoir au lendemain de la révolution de 1848 ils tentèrent de composer un programme de réforme pour des temps de transition qui fut en accord avec leur modèle.

Après une courte présentation du profil biobibliographique de ces trois auteurs (I) nous aborderons la part critique de leurs œuvres. Si, selon eux, le régime libéral de la Monarchie de Juillet produisait de tels fléaux - crises économiques, paupérisme, inégalités vertigineuses -, c'était, en raison de l'absence de tout principe directeur et du choix de ne pas gouverner, i.e, de laisser la société commandée par les hasards du fonctionnement aléatoire des marchés (II). Face à cette absence de choix, Blanc, Pecqueur et Vidal revendiquaient la nécessité de définir l'idéal ou l'utopie qu'ils concevaient comme une réflexion fondamentale sur la justice permettant, en aval, de guider la réforme sociale (III). Dès lors, il leur revenait de définir des principes de justices (IV) puis de signaler comment ces principes pouvaient permettre de concevoir les institutions d'une future société juste, mais aussi, au présent, de travailler à la réforme des institutions économiques $(\mathrm{V})$. Nous tenterons finalement en conclusion de proposer, prudemment, quelques rapprochements entre ces réflexions qui firent éclosion autour de 1848 et les théories modernes de la justice.

\footnotetext{
${ }^{1}$ Sur le plan méthodologique, cet article s'inspire librement des orientations proposée par l'histoire intellectuelle, telle que définie dans Skinner (2002) et qu'ont présentées récemment au public français, Spitz (1989), Guilhaumou (2006), Plon (2001), Gautier (2004).
} 
Le tournant des années 1840 marque une date charnière pour les socialistes français. En l'espace de quelques mois parurent plusieurs titres majeurs, Le Voyage en Icarie d'Etienne Cabet, Qu'est-ce que la propriété de Pierre-Joseph Proudhon et Organisation du travail de Louis Blanc; ces ouvrages inauguraient une seconde vague de contributions socialistes qui donnaient un nouvel élan et renouvelaient en partie une réflexion entamée au tournant des années 1830 par les saint-simoniens orthodoxes menés par les deux Pères, Barthélémy-Prosper Enfantin et Saint-Amant Bazard, les fouriéristes Victor Considérant, Jules Lechevalier ou Abel Transon, les dissidents (Pierre Leroux et Philippe Buchez) et quelques républicains (dont François-Vincent Raspail). En 1840, rebutée par l'échec des insurrections de 1834 et de 1839, influencée aussi par la parution des Paroles d'un croyant (1834) de Félicité de Lamennais enjoignant une nouvelle alliance entre le christianisme et la réforme sociale, la plus grande partie des écoles socialistes pouvaient se ranger sous la bannière de la fraternité, valeur cardinale de la République ${ }^{2}$. Nous focaliserons cette étude sur un groupe plus restreint composé de Louis Blanc (1811-1882), de Constantin Pecqueur (1801-1887) et de François Vidal (1812-1872). Deux raisons peuvent être avancées à cette limitation de l'étude à ces trois auteurs, l'une relevant de la pensée économique et sociale, l'autre relevant de l'engagement politique.

Sur le plan théorique, il est incontestable que ces trois auteurs s'influencèrent au moment de la publication de leurs principaux travaux ce qui leur permit, nous le verrons, de proposer un discours sinon identique, du moins commun. Pecqueur était l'aîné du groupe ; journaliste, il avait côtoyé les saint-simoniens du Globe en 1831-1832 puis était passé dans le camp des fouriéristes du Phalanstère, avant de s'éloigner de ce groupe et de se rapprocher encore des positions républicaines ${ }^{3}$. En 1837 il publiait un ouvrage important, à la fois synthèse de son cheminement dans les écoles socialistes du début des années 1830 et annonce des évolutions

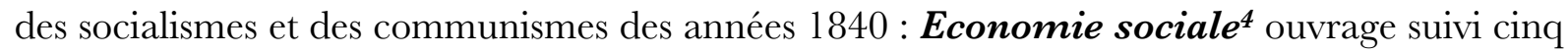
années plus tard d'un volume plus étoffé, Théorie nouvelle d'économie sociale et politique $^{5}$. Louis Blanc, plus jeune de dix ans, commençait véritablement sa carrière de publiciste en 1834 devenant très tôt l'un des principaux rédacteurs du journal républicain $\boldsymbol{L} \boldsymbol{e}$ Bon Sens $^{6}$. Rapidement devenu l'un des ténors du mouvement républicain, il créait en 1839 son propre journal, la Revue du progrès où paraissait sous forme d'articles L'Organisation $\boldsymbol{d u}$ travail. Publié sous forme d'ouvrage l'année suivante, le titre eu un énorme

\footnotetext{
2 Marcel David, Le printemps de la fraternité (1830-1851), Paris, Aubier, 1992; Andrea Lanza, All'abolizione del proletario! Il discorso socialista fraternitario, Parigi 1839-1847, Milano, Franco Angeli, 2010.

${ }^{3}$ La thèse ancienne de Gérard Marcy demeure encore l'une des rares références sur le sujet; G. Marcy, Constantin Pecqueur fondateur du collectivisme d'Etat, Paris, Sirey, 1934.

${ }^{4}$ Constantin Pecqueur, Economie sociale. Des intérêts du commerce, de l'industrie, de l'agriculture sous l'influence des applications de la vapeur, Paris, Capelle, 1839.

${ }^{5}$ Constantin Pecqueur, Théorie nouvelle d'économie sociale et politique ou Etudes sur l'organisation des sociétés, Paris, Capelle, 1842. Pecqueur publia tous ses ouvrages importants entre 1837 et 1844. En plus des ouvrages mentionnés ci-dessus, il publia notamment, Des Améliorations matérielles dans leurs rapports avec la liberté ; introduction à l'étude de l'économie sociale et politique, Paris, Gosselin, 1840 ; De la paix, de son principe et de sa réalisation, Paris, Capelle, 1842 ; De la République de Dieu, Union religieuse pour la pratique immédiate de l'égalité et de la fraternité universelle, Paris, Charpentier, 1844. A partir de 1845 il reprit sa pratique journalistique et fonda finalement son propre journal, Le Salut du peuple qui connut six numéros entre décembre 1849 et mai 1850.

${ }_{6}^{6}$ Sur Louis Blanc, et pour se limiter aux publications récentes; Françis Démier (dir.), Louis Blanc: un socialiste en république, Paris, Creaphis, 2005 ; Benoit Charruaud, Louis Blanc, La république au service $\boldsymbol{d u}$ socialisme, Thèse droit, université Robert Schuman, 2008.
} 
retentissement connaissant cinq éditions et plusieurs ajouts avant 1848, neuf éditions et de larges remaniements en 1850. En 1843, tout comme Pecqueur qui y signait les articles d'économie, Blanc rejoignait le journal La Réforme que venait de lancer Alexandre LedruRollin et où s'exprimaient alors les républicains radicaux favorables aux réformes économiques et sociales d'envergure ${ }^{7}$, les républicains qui organiseront la campagne des banquets en 1847, prélude à la révolution, puis prendront les rênes du pouvoir en février 1848. François Vidal participait à ce mouvement d'ensemble ${ }^{8}$ : proche au tout début des années 1840 de la rédaction du journal fouriériste La Démocratie pacifique, écrivant peu après dans la Revue indépendante (que coordonnaient Pierre Leroux et George Sand et où écrivait également assidument Pecqueur) il rédigeait d'abord une courte brochure sur les caisses d'épargne, avant de publier en 1846 un large volume, De la répartition des richesses ou De la justice distributive en économie sociale. En 1850, Constantin Pecqueur évoquera donc pour désigner ce groupe ayant réussi la synthèse du saint-simonisme, du fouriérisme et du communisme « les Socialistes unitaires, fraternels et égalitaires (Pecqueur, $1850: 14)$.

Au lendemain des journées révolutionnaires de 1848, la présence de Louis Blanc (ainsi que celles de Ferdinand Flocon ou de «l'ouvrier Albert») était imposée par les travailleurs au sein d'un gouvernement provisoire dirigé par Jacques Dupont de l'Eure et dominé par les républicains modérés attentifs en priorité à la réforme politique, soucieux surtout de ne pas se laisser déborder par les revendications sociales. Désireux de voir se réaliser la création d'un ambitieux ministère du progrès où serait expérimentée à vaste échelle l'organisation du travail expression de la revendication du droit au travail, Louis Blanc se voyait débouté par ce gouvernement. Le ministère était remplacé par une commission consultative sans budget propre qui à partir du $1^{\text {er }}$ mars allait siéger dans le lieu symbolique du Palais du Luxembourg (lieu auparavant des assemblées des Pairs de France). Dans l'objectif de faire vivre cette institution improbable, Blanc, nommé président de la Commission s'entourait d'un viceprésident, Albert mais surtout de Pecqueur et Vidal' ${ }^{9}$. Dans l'espace de ses deux mois d'existence, la Commission allait innover triplement :

d'abord, pensée comme un véritable (et inouii) « parlement du TRAVAIL » ${ }^{10}$, la Commission «appelait pour la première fois le Peuple à la discussion publique de ses intérêts ${ }^{11}$. Elue par les différentes corporations de métiers caractéristiques surtout de l'artisanat urbain parisien, une représentation de dix travailleurs allait siéger et participer de concert avec une représentation patronale aux séances du Luxembourg. Attentif aussi à faire entendre dans le cadre de cette vaste "discussion publique ${ }^{12}$, les différents pans de l'opinion savante, Blanc avait également prévu une commission d'experts qui allait permettre à des économistes aussi divers que Louis Wolowski, Charles Dupont-White, Frédéric Le Play, Victor Considérant, Jean Reynaud de participer aux discussions générales.

Ensuite, la Commission s'attacha aux mesures urgentes. Des réformes furent discutées et négociées concernant la durée du travail ou les marchandages toxiques, et, surtout, un système de conciliation des différends industriels entre ouvriers et patrons (sorte de

\footnotetext{
${ }^{7}$ L'autre grand journal républicain, Le National, se focalisait sur les réformes politiques.

8 On dispose de très peu d'informations biographiques sur Vidal; quelques maigres informations dans Maisonneuve (1898) ainsi que dans la notice qui lui est consacrée dans le Dictionnaire des parlementaires français.

${ }^{9}$ Dans ses souvenirs sur 1848, Blanc écrit pour souligner «l'inappréciable appui que me prêtèrent M. Vidal, secrétaire général de la Commission, et M. Pecqueur, tous les deux hommes d'un mérite éminent, d'un vaste savoir, et profondément versés dans la science de l'économie politique », Louis Blanc, Histoire de la révolution de 1848, Paris, Flammarion, 1880, vol. 1, p. 168.

${ }^{10}$ Id. p. 168

${ }^{11}$ Id., p. 137.

12 Id., p. 147.
} 
reproduction à vaste échelle de la logique (idéalement) paritaire des conseils des prud'hommes) fut instituée. Comme le signalait ultérieurement Blanc, dans ce «tribunal arbitral... des conciliations sans nombre furent opérées ${ }^{13}$ concernant les paveurs, voituriers, couvreurs, mécaniciens, cochers, boulangers...

Enfin, la Commission tenta d'adosser les réformes en cours à un plan d'ensemble, un programme doctrinal, présenté auparavant par Blanc, Vidal et Pecqueur dans leurs ouvrages des années 1840. Selon une double logique (que nous détaillerons en section V), une partie de l'action consista à impulser la création d'ateliers sociaux, des associations coopératives, qui, effectivement furent expérimentés par les tailleurs, puis par les selliers, les fileurs, les ébénistes et d'autres corporations encore. Simultanément, un plan de transformation sociale d'ensemble fut rédigé par les trois hommes, rappelant la nécessité des ateliers sociaux (industriels et urbains), mais aussi complétant le programme par l'établissement de colonies agricoles (sur le modèle coopératif) et par des réformes d'envergure mettant l'Etat au cœur de l'économie en lui donnant la responsabilité d'administrer le crédit et la banque, les assurances, et le commerce. Publiée dans plusieurs livraisons du Moniteur du début du mois de mai 1848 cet Exposé de la doctrine du Luxembourg fut repris et explicité dans l'ouvrage de Vidal Vivre en travaillant. Projets, voies et moyens de réformes sociales (1848) et conduisit Blanc à remanier presque entièrement la neuvième édition de Organisation du travail (1850).

L'aventure du Luxembourg fut stoppée par les journées du 15 mai et surtout celles des 22-26 juin 1848. Elu à l'Assemblée nationale aux élections du 23 avril 1848 (les premières à appliquer le suffrage universel), Blanc que l'on créditait injustement de l'expérience des ateliers nationaux (organisée par Marie et le gouvernement provisoire en grande partie contre l'expérience des ateliers sociaux du Luxembourg) était accusé d'être à l'origine de l'émeute du 15 mai (envahissement de l'Assemblée par une émeute populaire) et devait partir pour un exil en Angleterre qui durera 22 ans. Vidal, fondateur en janvier 1849 du journal Le Travail affranchi, sera élu député (Montagne et minorité démocratique) de la Seine aux élections complémentaires de mars $1850^{14}$. Pecqueur, nommé bibliothécaire de l'Assemblée nationale, créait en décembre 1849 son propre journal, Le Salut du peuple. Après la proclamation de l'Empire, en décembre 1851, les deux hommes partent en exil intérieur dans leurs régions respectives.

II.

\section{Dénonciation du régime de concurrence}

Lorsque Organisation du travail parut sous forme d'ouvrage au tout début des années 1840, les 2/3 du volume étaient consacrés à démontrer que le régime actuel de concurrence constituait un "système d'extermination » pour le peuple, une "cause de ruine pour la bourgeoisie », et un ferment de « guerre à mort » entre les nations européennes. Lors de ces années charnières, chacun des trois socialistes fraternitaires allait investir un terrain d'enquête privilégié révélant les manifestations pathologiques de la libre concurrence. Historien du temps présent, Louis Blanc fera des révoltes ouvrières lyonnaises de 1831 et 1834 l’indice des fléaux engendrés par le libéralisme des notables mis en place par la Monarchie de Juillet ${ }^{15}$. Envoyé en 1839 par Jules Faure (ministre des travaux publics) étudier le système belge des chemins de fer, qui s'appuyait sur l'Etat et le corps restructuré des ingénieurs civils, Pecqueur

\footnotetext{
${ }^{13}$ Id., p. 184.

14 Note sur les deux ouvrages publiés par Vidal après 1850.

${ }^{15}$ Louis Blanc, Histoire de dix ans 1830-1840, tome 3 (chapitre 2) et tome 4 (chapitre 5), Paris, Pagnerre, 1842-1844.
} 
fit de cette enquête un réquisitoire contre le système des concessions privées qui avait les faveurs des parlementaires français, dénonçant dans ces concessions, une «monstruosité sociale et économique $»^{16}$. Enfin Vidal stigmatisera dans ses premiers travaux la féodalité économique et financière que favorisait l'organisation présente du crédit et de la banque en France. Qu'entendaient ces Socialistes Fraternitaires par «régime de concurrence »? Il est important de constater que leurs arguments ne portaient pas sur la forme des marchés, car leurs attaques s'adressaient aussi bien aux marchés de concurrence qu'aux monopoles industriels. Leurs critiques atteignaient donc aussi bien les économistes partisans du libéralisme économique que certains hommes du gouvernement de Juillet, Adolphe Thiers ou Charles Dupin par exemple, partisans du protectionnisme, défenseurs des grands intérêts économiques des notables et des lobbys en formation dans les domaines du chemin de fer, de l'agriculture et de la banque. De fait, la critique des Fraternitaires portait essentiellement contre l'Orléaniste, c'est-à-dire ici une doctrine qui avait fait la suture entre le libéralisme politique conservateur d'un François Guizot et le libéralisme économique radical d'un Charles Dunoyer $^{17}$. En 1840 dans son pamphlet Réforme électorale ${ }^{18}$, Pecqueur signalait aux travailleurs «que sont les électeurs, les députés et les pairs? Ce sont précisément ces hommes qui, sous l'habit de bourgeois et en qualité d'entrepreneurs d'industrie, de capitaliste et de propriétaires fonciers, vous tiennent à leur solde, vous donnent ou vous retirent arbitrairement le travail, font pour vous la pluie et le beau temps, vous marchandent vos salaires, augmentent impitoyablement la longueur de vos journées et celles de vos jeunes enfants, qui se coalisent tacitement pour mieux mettre vos bras au rabais » p. 30. De façon synthétique, Blanc notait que, « la concurrence, c'est la guerre transportée dans le domaine du travail » (LCS : 221). La concurrence était donc proprement un scandale, introduisant la logique de la guerre, domination et destruction, dans un domaine, le travail où devait régner coopération et production. Et la concurrence désignait de façon générique pour ces Socialistes toute relation économique non contrôlée par un pouvoir politique représentatif de l'ensemble des intérêts concernés.

Ce régime de concurrence posait, selon ces trois socialistes fraternitaires, un double problème, d'efficacité et d'équité.

La concurrence se révélait être un processus inefficace. L'équilibre entre l'offre et la demande ne pouvait être réalisé dans un système sans régulation, et la concurrence produisait donc crises périodiques, mauvaise orientation des efforts et gaspillages des ressources. Par ailleurs, le système était instable, car il situait l'antagonisme au cœur des transactions économiques et sociales. Dès lors en raison des déséquilibres de pouvoir entre les agents, la concurrence conduisait inéluctablement à la constitution de monopoles faisant primer la rentabilité sur l'efficacité, les intérêts d'une minorité d'individus, sur la richesse collective de la société. Enfin, système sans prévision et à courte vue, le régime de concurrence ne favorisait pas la gestation des innovations majeures qui devaient scander l'évolution sociale.

La concurrence était également synonyme d'iniquité. Loin d'être un phénomène pur, indépendant des autres phénomènes sociaux, l'échange concurrentiel intervenait dans un environnement institutionnel que dominait la propriété individuelle et héréditaire. Dans ce contexte l'antagonisme que la concurrence plaçait au cœur des relations sociales mettait en présence des individus et des groupes inégalement dotés en richesse et

\footnotetext{
${ }^{16}$ Constantin Pecqueur, De la Législation et du mode d'exécution des chemins de fer, par C. Pecqueur ; lettres adressées à M. le Ministre des Travaux publics, Paris, Desessart, 1840, p. VII.

${ }^{17}$ Sur cette doctrine, voir principalement Rosanvallon (1985, 2011).

${ }^{18}$ Constantin Pecqueur, Réforme électorale : Appel au peuple à propos du rejet de la pétition des 24000, Paris, Desessart, 1840. La révolution de 1830 avait modifié, mais de façon étroite les règles de la citoyenneté politique, fixant le cens électoral à 200 francs et le cens d'éligibilité à 500 francs. Ces restrictions qui ne furent pas modifiées jusqu'à 1848 limitaient les citoyens politiques à 240000 individus dans un pays comptant alors 32 millions d'habitants.
} 
surtout en pouvoir, des propriétaires et des non-propriétaires. La situation était inacceptable car, l'origine des dotations (et leur accumulation ultérieure) n'était pas justifiée ; car les inégalités ne pouvaient, dans un contexte non régulé, que s'accentuer touchant par exemple des catégories de plus en plus nombreuses (les bourgeois et les classes moyennes bientôt menacés de «ruine» par les «oligarques industriels»); car, enfin, ce jeu déséquilibré conduisait dès à présent à des conséquences sociales et morales extrêmes (« l'extermination » des pauvres et la généralisation de «l'exploitation de l'homme par l'homme ») que toute société civilisée ne pouvait que refuser: misère, paupérisation, prostitution, corruption, criminalité.

Cette critique de la concurrence proposée par Blanc, Vidal ou Pecqueur se situait dans le prolongement de celle présentée depuis une décennie par les différentes écoles socialistes, au moins depuis Exposition de la Doctrine de Saint-Simon (1828-1829). Elle était d'ailleurs étroitement associée à une critique de la propriété individuelle et héréditaire. Mais ce qui était plus distinctement reproché par les Socialistes Fraternitaires aux thuriféraires du régime de concurrence était leur attitude générale de capitulation face au projet de gouverner la société et son évolution. Les économistes de l'Ecole de Jean-Baptiste Say étaient ainsi systématiquement critiqués car leur doctrine les conduisait à capituler devant les faits, et à défendre, comme le soulignait Pecqueur, «l'idée monstrueuse que tout ce qui est est bien » (1839: p. 199). Cela revenait à une reddition devant le hasard, le «Dieu hasard» (CP, Réforme électorale, p. 76), Blanc dénonçant par exemple, «l'humiliante dictature du hasard » (Droit au travail, 1849 : p. 25) et Vidal, plus précis dénonçant «la théorie transcendante de la force et du hasard» (1848 : 256-257). Et ce hasard aboutissait non à la providence, mais à la domination et à l'exploitation exacerbée de l'homme par l'homme. Une telle capitulation était possible chez ces auteurs en raison de leur incroyance. S'ils s'en remettaient aux hasards et aux forces des marchés, c'est qu'ils ne croyaient pas en l'existence de principes rationnels ou même simplement raisonnable de gouvernement des sociétés. Or, l'objectif de Blanc, Pecqueur et Vidal était de souligner que ces idées, ces principes existaient, et qu'il était en outre possible de les mobiliser pour organiser une société soumise jusqu'alors aux lois de l'antagonisme et du chaos. Le programme général des Fraternitaires pouvait alors être résumé en quelques mots: la critique de l'inorganisation économique et politique présente devait conduire à une réflexion fondamentale et préalable sur la justice: Vidal précisait, «nous chercherons théoriquement le principe de justice qui devrait présider à la répartition des richesses... nous indiquerons sommairement les moyens à prendre pour remédier au désordre économique, pour contrebalancer les funestes effets des institutions existantes » alors que, de son côté, Pecqueur annonçait que «toutes les relations humaines, toutes, jusqu'aux plus immatérielles, pivotent plus ou moins directement sur ce grand fait: la DISTRIBUTION DES RICHESSES » (Réforme électorale, p. 114).

\section{Rêveurs et utopistes dans des temps de transition}

Les Socialistes Fraternitaires estimaient que la société de leur temps souffrait essentiellement d'un déficit d'intelligence sociale. C'est ce déficit qui conduisait à abandonner son évolution aux lois du hasard et de la force et à le payer au prix fort par l'aberration que constituait en régime de concurrence la croissance parallèle de l'industrie et du paupérisme : cette allure aberrante (plus encore qu'aveugle) de l'évolution était contraire à l'idée de progrès qui caractérisait les Fraternitaires comme toute cette génération de penseurs réformistes. Face à cette faillite, ils réhabilitaient le rôle des idées comme guides indispensable de toute action. Face à la glaciation des esprits, Blanc prônait les « rêveries » et autres «témérités de l'esprit » 
(1841 : 72), alors que Vidal défendait les «idées systématiques » et ajoutait que les socialistes : « se préoccupent de l'idéal, du juste, du vrai, du possible, bien plus que du réel ; et c'est par la transformation des idées qu'ils veulent arriver à modifier les institutions, à exercer une action » (1846 : 12-13). Pour sa part, dès l'introduction de sa Théorie nouvelle d'économie sociale, Pecqueur annonçait « rien de plus capital à nos yeux que cette exploration de l'idéal et même de l'utopie », expliquait qu'il se ferait le partisan « des vrais principes, des utopies si mal famées auprès des retardataires » et qu'il cheminerait en compagnie de «cette noble phalange d'Utopistes » $(1842: \text { I })^{19}$.

Le fait de revendiquer ostensiblement la qualification infamante d'utopiste que ne cessaient de leur accoler leurs adversaires du «juste-milieu » n'était pas anodin en 1840 et signalait le degré de conviction qu'avaient ces Socialistes Fraternitaires dans l'objectivité et l'effectivité des principes et des idées qu'ils tentaient de découvrir. Selon eux ces caractéristiques étaient attestées à la fois par une histoire et par un code.

Dans les premières phrases de Vivre en travaillant, Vidal avouait, "le germe des théories que propagent aujourd'hui les utopistes les plus avancés, se trouvent déjà dans les livres qui ont été écrits il y a plus de deux mille ans, dans des livres dont les auteurs sont restés inconnus (1848: 21). La validité des principes travaillés par Blanc, Vidal et Pecqueur était d'abord prouvée par le fait qu'ils avaient été précédemment tâtonnés et approximés par d'anciennes et puissantes traditions intellectuelles et que cette enquête signalait un progrès. Les Socialistes Fraternitaires revendiquaient pour ces principes une triple filiation. Celle du socialisme de 1830, héritier lui-même des expériences pionnières de Charles Fourier ou de Saint-Simon, et qui avait mis à jour le problème de la propriété, souligné la nécessité de l'association, proposé de croiser la question politique (la République) et la question sociale. Celle, en amont, de la Révolution française, 1793 et ses idées d'organisation et d'égalité plus encore que 1789 et sa définition insuffisante de la liberté. Celle, en amont encore, du Christianisme originel et de ses croyances sur la pauvreté, la propriété, le partage, la communauté et, enfin et surtout, l'égalité. Dévoilant ainsi ses sources intellectuelles, Pecqueur précisait: "Nous répondrions : Jésus-Christ, Rousseau, toute la Révolution française, SaintSimon, Fourier: - Jésus-Christ et toutes les Bibles pour la formule fondamentale de la morale; pour la face spirituelle du problème économique ; le Saint-Simonisme pour la face sociale, gouvernementale et historique ; - Fourier pour la face matérielle de l'association et de la solidarité ; - Rousseau et la Révolution française pour le côté politique et l'esprit d'égalité et de liberté » (1842: IV).

Dans l'esprit des Socialistes Fraternitaires, les idées n'étaient pas que la résultante d'une histoire ou d'une trajectoire. Leur objectivité et leur effectivité venaient surtout du fait qu'elles étaient l'expression d'un code. Vidal, qui mobilisait l'idée de destinées, et surtout Pecqueur reprenaient en lui donnant une extension nouvelle l'idée fouriériste d'un code divin donnant la clé de l'organisation sociale. Les Fraternitaires bénéficiaient ici à la fois des clarifications antérieures du fouriérisme réalisées dès 1833-1834 par l'équipe du journal Le Phalanstère (une équipe à laquelle avait appartenu Pecqueur) et par la parution des ouvrages de PierreSimon Ballanche, Philippe Buchez ou Félicité de Lamennais qui (parmi bien d'autres) avaient

\footnotetext{
${ }^{19}$ Le fait de localiser les idées au cœur de la réforme reflétait aussi l'espoir placé par ces hommes sensibles au développement de la discussion publique dans la naissance de la presse moderne, nouveau media support d'une révolution communicationnelle depuis le début des années 1830 en France. Dans son texte sur la réforme politique, Pecqueur signalait : «la guerre par la presse est tout aussi décisive pour le progrès de la civilisation que les plus fameuses batailles, et elle est moins sanglante... Il est donc une guerre qui peut se faire en temps de paix, guerre qui doit être funeste à tous les privilèges sans exception ; c'est celle des idées » (Pecqueur, Réforme électorale, p. 73).
} 
définitivement imposés l'idée des affinités électives entre christianisme et progrès ${ }^{20}$. En 1849, Pecqueur expliquait que le peuple ne pourrait accéder au salut que «par la connaissance, l'amour et la pratique des lois et des conditions mises par Dieu à l'harmonie universelle » (1849 : 10). Toutefois, sans que la contradiction soit réglée ou même ouvertement affrontée, ces mêmes auteurs, Blanc le premier, s'appuyaient sur la tradition des Lumières pour faire de ces idées l'expression de la raison humaine, raison individuelle éclairée par l'instruction, mais aussi raison collective résultant de la pratique de la «discussion publique ». Enfin, il faut ajouter que, sur ce chapitre, les positions des trois auteurs, elles-mêmes évolutives, ne se superposaient pas exactement. Il est clair, en effet, que Pecqueur était sans doute le plus enclin à rechercher plutôt un fondement d'abord théologique à ces idées sur éthique et justice, alors que Blanc recherchait plus volontiers ces fondements dans une anthropologie philosophique, Vidal occupant une position intermédiaire entre les deux hommes.

Les Socialistes Fraternitaires revendiquaient donc leur intention de frayer la voie des idéaux et des utopies. Ces idéaux existaient et leur application traçait la voie rectiligne du progrès ; mais il était de la responsabilité des hommes d'appliquer ou non et plus ou moins fidèlement ces principes, et cela expliquait que l'histoire pouvait adopter des rythmes propres. Et les Fraternitaires ne faisaient pas mystère du caractère de ces utopies qui devaient, au premier chef, permettre de répondre à la question, Qu'est-ce qu'une société juste ? et à en tirer les conséquences en matière de réforme ; «je cherche des principes de justice distributive, universel et immuable » écrivait ainsi Pecqueur (1842:2), « nous allons sonder ce redoutable mystère de la justice distributive » lui faisait écho Vidal quatre ans plus tard (1846: 9) résumant ainsi l'intention théorique première de ce courant.

\section{Les principes d'une société juste}

Pour les Socialistes Fraternitaires, une société était juste lorsqu'elle conduisait au bonheur, une notion dont Vidal donnait la formule synthétique suivante : «le bonheur, pour l'individu, pour l'espèce, c'est la satisfaction complète de tous les besoins naturels, et par conséquence légitimes, moraux et physiques ; c'est, de plus, le développement progressif et harmonique de toutes les facultés, de tous les éléments qui constituent la nature humaine » (1846:22). Le bonheur, c'était d'abord la négation du malheur qu'annonçaient toutes les doctrines sur la résignation. Le bonheur recelait l'idée d'un pouvoir étendu des hommes correctement organisés sur le milieu naturel et social. Il fallait donc remettre sur le métier la tâche définie une première fois en 1825 par Saint-Simon dans Le nouveau christianisme : "le vrai progrès social dans l'histoire générale, renchérissait Pecqueur, est synonyme d'une grande amélioration dans le sort de la classe la plus nombreuse et la plus pauvre » (Réforme électorale 1840, p. 65).

Or, une société ne pouvait alors être juste et conduire au bonheur qu'à deux conditions : il fallait d'une part que les valeurs de liberté, d'égalité et de fraternité, correctement redéfinies, y soient chacune respectées; il fallait, d'autre part, que ces trois valeurs soient simultanément présentes, et correctement articulées entre-elles.

Liberté. Les Socialistes Fraternitaires accusaient 1789 d'inconséquence sur le chapitre de la liberté. La liberté individuelle, la liberté bourgeoise reconnue comme un droit en 1789, ne faisait en réalité que substituer une tyrannie nouvelle, celle des propriétaires, à une tyrannie ancienne, celle des rois et des aristocrates. Dans son style apocalyptique, Pecqueur dénonçant

\footnotetext{
20 Mentionnons ici P. S. Ballanche, Essais de palingénésie sociale, Paris, Didot, 1827 ; P. Buchez, Introduction à la science de l'histoire, ou Science du développement de l'humanité, Paris, Paulin, 1833 ; F. Lamennais, Paroles d'un croyant, Paris, Eugène Renduel, 1834 ; sur cet essor, voir Franck Paul Bowman, Le Christ des barricades 1789-1848, Paris, Cerf, 1987 et Edward Berenson, Populist Religion and LeftWings Politics in France 1830-1852, Princeton, Princeton University Press, 1984.
} 
l'emploi sans retenues que les économistes faisaient de cette notion de liberté-droit, pouvait noter, «la liberté illimitée, c'est le chaos ; c'est la sauvagerie, c'est l'anthropophagie » (SP, fev. 1850 : 6). A la notion de droit conféré à l'individu, il fallait substituer la notion de pouvoir garanti par la société ; "avec le mot droit, écrit Blanc, la liberté n'est qu'une théorie vague, tandis que le pouvoir tend à en faire une chose réelle» (CS: 4). Il pouvait donc proposer la définition suivante de la liberté : «la liberté consiste, non pas dans le droit, mais dans le pouvoir donné à chacun de développer ses facultés » (RFL : 77), ce que Pecqueur traduisait en soulignant, "absolument, être libre, c'est pouvoir ce qu'on veut» (SP). La liberté réelle, la «liberté intelligente» (Pecqueur) consistait donc en un ensemble de conditions morales et physiques que les institutions d'une société garantissaient également à tous les individus, « la jouissance sociale des conditions normales [du] développement moral et physique, à l'indéfini » (SP 1850). Les Fraternitaires modifiaient donc le discours ambiant sur l'idée de capacité naturelle des individus qui se trouvait, sous des formes différentes dans la France des années 1830-1840, aussi bien dans le libéralisme élitaire d'un François Guizot que dans le projet de société industrielle hiérarchisée des saint-simoniens orthodoxes. Il n'était pas tant chez Vidal, Pecqueur ou Blanc nécessaire de faire émerger une élite capable de configurer rationnellement et hiérarchiquement la société (et de placer chaque individu à sa place dans l'atelier social) que de rendre par des garanties sociale en termes de travail ou d'éducation tous les individus capables de fonctionner et de participer ainsi, chacun à sa mesure, au développement de la société.

Egalité. Dans son ouvrage sur la justice distributive, Vidal commençait par faire la recension critique des théories des économistes, des Physiocrates aux élèves de Say, puis il effectuait un passage en revue des positions des différentes écoles socialistes. Dans leur logique capacitaire, ("à chacun selon ses capacités, à chaque capacité selon ses œuvres »), les saintsimoniens privilégiaient une étroite proportionnalité entre contribution et rétribution, alors que, pour leur part, les fouriéristes proposaient de rémunérer en proportion le travail, le capital et le talent. Raisonnant en termes de «justice absolue », Vidal donnait lui sa préférence aux socialistes de l'école « égalitaire », notamment bien sûr Blanc et Pecqueur, qui avaient choisis comme principe de distribution « de la bonne volonté manifestée et des besoins de l'individu » (1846: 452). Dans les dernières lignes de l'Organisation du travail, Louis Blanc indiquait que même en cours de réforme et de réorganisation de la société par les associations et par l'Etat, l'état des esprits et le niveau d'éducation des individus devaient laisser subsister longtemps un certain niveau d'inégalité. Toutefois, il ajoutait que les principes purs de justice distributive devaient, dans une société future complètement organisée, faire disparaître ces écarts et conduire à l'égalité en matière de distribution des salaires et des bénéfices. Il écrivait, « le jour viendra où il sera reconnu que celui-là doit plus à ses semblables qui a reçu de Dieu plus de force ou d'intelligence. Alors il appartiendra au génie, et cela est digne de lui, de constater son légitime empire non par l'importance du tribut qu'il lèvera sur la société, mais sur la grandeur des services qu'il lui rendra. Car ce n'est pas à l'inégalité des droits que l'inégalité des aptitudes doit aboutir, mais à l'inégalité des devoirs ». Répondant un peu plus tard aux critiques de Michel Chevalier, il insistera, "si la hiérarchie par capacité est nécessaire et féconde, il n'en est pas de même de la rétribution par capacité » (1847: 141). Pecqueur expliquait lui aussi que dans une société parfaitement organisée, «chacun remplissant convenablement les devoirs de sa fonction, tous, sans exception, ont droit à l'égalité de rétribution ou de part dans le produit et dans les avantages sociaux »; en effet, poursuivait-il, "l'égalité consiste pour chacun à obtenir par rapport à tous l'équivalente satisfaction de ses besoins fondamentaux. Cette équivalence est toujours possible, car les besoins fondamentaux de la vie sont à-peu-près les mêmes dans tous les individus » (1842: 667-668). La règle distributive de ces socialistes était donc« de chacun selon ses forces et ses aptitudes; à chacun selon ses besoins » (Pecqueur, SP, décembre 1849, p. 4] ou « De 
chacun selon ses facultés, à chacun selon ses besoins » (Blanc, LCS, p. 224). Là encore, la position des Fraternitaire conduisait, dans l'idéal d'une théorie pure de la justice distributive, à renverser les discours ambiant sur la relation entre capacité et rétribution. En effet, logiquement, et cette position sera adoptée par Blanc, une société juste devait concevoir que c'étaient les faibles qui avaient le plus de besoins et nécessitaient le plus de garanties sociales pour leur développement. Il fallait donc envisager le fait que les distributions les plus élevées aillent aux individus les moins capables.

Fraternité. Chez les Socialistes Fraternitaires, libertés et égalités étaient des principes qu'assuraient, que garantissaient aux individus les institutions d'une société organisée. La fraternité, comme principe, renvoyait plus chez ces auteurs à la contrepartie, en termes de comportement, que les individus devaient alors à cette société organisée. Si Blanc expliquait métaphoriquement que la fraternité correspondait à «l'égalité consacrée, poétisée, sanctifiée et maintenue par l'amour»(LCS), Pecqueur adoptait une position plus avancée : «la fraternité seule est le principe social générateur par excellence. L'égalité et la liberté délimitent et précisent la fraternité ; elles en sont en quelque sorte le poids et la mesure. Sur l'égalité et la liberté on ne bâtit que l'égoïsme, le droit et le fédéralisme: sur la fraternité on greffe le dévouement, le devoir et l'unité » (1842: 3). On retrouve à ce niveau une différence de perspective entre les deux hommes, l'un, Pecqueur, recherchant plus nettement les fondements de ses idées sur justice et éthiques sociales dans la théologie, l'autre, Blanc, dans une anthropologie philosophique; cela n'empêchant d'ailleurs pas les deux hommes de piocher dans le registre d'en face, le socialisme étant par exemple pour Blanc «l'évangile en action » (LCS : 3) ; mais il y avait là, on l'a dit, une constante de cette génération de penseurs réformistes qui entremêlaient de mille façons le religieux et le politique. Néanmoins une différence demeurait. Pour Pecqueur la fraternité était rectrice car le devoir découlait de Dieu, il est «l'obligation de faire la volonté de Dieu », le droit étant, "tout ce que l'homme a la liberté de faire ou de ne pas faire, sans contrarier la volonté de Dieu, ou plutôt remplir son devoir » (1842: 21 $)^{21}$. Pour Blanc, si la fraternité n'était «qu'une exposition poétique de la solidarité » (R1848 : 145) c'était parce que ce c'étaient d'abord les conditions institutionnelles réalisant la liberté et l'égalité qui façonnaient les comportements et permettaient la réalisation de la fraternité. Pour Blanc, les institutions d'une société organisée permettaient, en effet, de révéler graduellement le propre du comportement de l'homme. L'intérêt en constituait un ingrédient important, mais bien moins que le dévouement. Or, l'organisation induisait comme activité dominante le gouvernement, et, comme le résumera à plusieurs reprises Blanc lors de ses discours devant l'Assemblée ouvrière du Luxembourg, "gouverner c'est se dévouer » (RFL). Sur ce dernier point, une nouvelle fois, Blanc était débiteurs des doctrines de son temps, cette idée de dévouement provenant, d'un côté, d'une source républicaine classique attentive aux vertus du citoyen, d'un autre côté, d'une source religieuse et du dévouement chrétien salué alors par des auteurs comme Buchez ou Lamennais.

Les Socialistes Fraternitaires définissaient donc leurs principes de justice à partir des trois éléments retravaillés de la devise républicaine. Deux éléments renvoyaient plus nettement à une logique d'institution, le troisième à une logique de comportement. Même si quelques hiérarchies entre les trois termes pouvaient se révéler chez tel ou tel auteur, le point crucial dans le cadre de leur conception générale de la justice était qu'il fallait que les trois principes soient simultanément respectés.

\section{Les institutions d'une société juste : Associations et Etat}

\footnotetext{
${ }^{21}$ Critiquant en 1850 la position de Proudhon, Pecqueur expliquait que « le pivot de l'unité sociale, c'est Dieu, le sentiment et l'idée religieuse », et il développait quelques pages plus loin plusieurs paragraphes sur l'infériorité du raisonnement par rapport à ce sentiment (SP, janvier 1850, p. 44 et suiv.).
} 
Au tournant des années 1840, les observateurs sociaux, Louis-René Villermé, Eugène Buret, Antoine-Honoré Frégier, avaient révélés dans leurs grandes enquêtes sociales l'ampleur du paupérisme et de la misère des classes laborieuses ${ }^{22}$. Pour le pouvoir orléaniste, la situation n'était pourtant qu'une affaire de comportement taré et débile des prolétaires, imprévoyants, irresponsables, inefficaces. Charles Dunoyer, économiste et idéologue du régime de Juillet expliquait alors que «l'effet du régime industriel est de détruire les inégalités factices; mais c'est pour mieux faire ressortir les inégalités naturelles » et il poursuivait, "il est bon qu'il y ait dans la société des lieux où soient exposées à tomber les familles qui se conduisent mal, et d'où elles ne puissent se relever qu'à force de se bien conduire. La misère est ce redoutable enfer. G'est un abîme inévitable, placé à côté des fous, de dissipateurs, des débauchés, de toutes les espèces d'hommes vicieux, pour les contenir $»^{23}$. Prenant le contre-pied de cette position qu'ils jugeaient scandaleuse, les Socialistes fraternitaires expliquaient que la misère qui touchait les $9 / 10^{e}$ de la population n'était pas la conséquence fatale d'un processus naturel de sélection des comportements adaptés aux transactions de l'industrie et du commerce, mais l'effet d'institutions défectueuses. Pecqueur expliquait ainsi que «le mal est moins dans les hommes que dans les institutions » (SP, $\left.\mathrm{n}^{\circ} 1, \mathrm{p} .7\right)$ et Blanc écrivait: "On accuse de tous nos maux la corruption de la nature humaine: il faudrait en accuser le vice des institutions sociales » (1847 : 179). Une société juste devait réaliser les principes de justice, mais cela demandait, comme le soulignait Vidal " une transformation radicale des institutions qui nous régissent » (Vidal, 456). Le régime de concurrence devait dès lors être entièrement transformé par l'intervention de deux institutions, l'association et l'Etat. Vidal, Blanc et Pecqueur avaient chacun tâtonné tout au long des années 1840 pour avancer dans l'identification théorique de ces deux institutions, dans la définition de leur fonction, et enfin dans la façon dont il fallait concevoir leur complémentarité. Une synthèse de leurs positions respectives fut présentée au printemps 1848 lors des discussions, discours et exposés de la Commission du Luxembourg ${ }^{24}$.

La Commission, rappelait-ils, avait dû agir dans l'urgence, et des mesures immédiates avaient été prises « dans l'orageuse ivresse d'une révolution ». Mais son but était également de « rassembler des matériaux pour l'avenir » (87), de proposer des orientations permettant de transformer « un ordre social vicieux » coupable simultanément de crises de surproduction et de paupérisation des masses. Ils expliquaient alors: "Or, deux grandes formes ou combinaisons semblent destinées à envelopper en quelque sorte les nouveaux rapports sociaux.... D'une part, l'association, principe de toute force et de toute économie; d'autre part, l'intervention désintéressée de l'Etat, principe de tout ordre, de toute justice distributive et de toute unité » (89). Ils ajoutaient encore qu'il fallait une réforme d'ensemble, un plan global et coordonné de transformation : "l'atelier social AGRICOLE et l'atelier D'ECHANGE, DE VENTE OU D'ACHAT, doivent être organisés en même temps que l'atelier social INDUSTRIEL » (91). Le plan de réforme comprenait donc deux volets assez distincts : « d'une part, des ateliers sociaux d'agriculture et d'industrie à organiser sur les bases nouvelles de

\footnotetext{
${ }^{22}$ L. R. Villermé, Tableau de l'état physique et moral des ouvriers employés dans les manufactures de laine, de coton et de soie, Paris, Renouard, 1840; H. A Frégier, Des classes dangereuses de la population dans les grandes villes et des moyens de les rendre meilleures, Paris, Ballière, 1840 ; E. Buret, De la misère des classes laborieuses en Angleterre et en France, Paris, Paulin, 1840.

${ }^{23}$ Charles Dunoyer, De la liberté du travail, t.1, Paris, Guillaumin, 1845, p. 345 ; Des objections qu'on a soulevé ces derniers temps contre le régime de la concurrence, Batignolles-Monceaux, Desrez, 1841, p. 38 .

${ }^{24}$ Edité dans le Moniteur du mois de mai, L'Exposé général de la doctrine du Luxembourg ne présente qu'une partie du programme. Pour compléter cet exposé, il faut, d'une part, vérifier les autres documents transmis par Louis Blanc dans son recueil La Révolution de février au Luxembourg. Il faut aussi voir l'exposé présenté en mai-juin 1848 par François Vidal, Vivre en travaillant....
} 
l'association et de la solidarité; de l'autre, des institutions à fonder, à modifier ou à transformer » $(93)$.

Le premier volet correspondait aux orientations sur les ateliers sociaux présentées par Louis Blanc dans l'Organisation du travail ; il tentait également d'y fondre les perspectives imaginées par Vidal sur les colonies agricoles. Le plan, précisait ultérieurement Blanc « consistait simplement à jeter, au milieu du système social actuel, les fondements d'un autre système, le système coopératif, en donnant au dernier le caractère d'une grande expérience nationale faite avec l'aide et sous le contrôle de l'Etat»(R1848 : 164). Dans cette vision l'institution transformationnelle était d'abord l'association que Blanc imaginait sous la forme d'une vaste coopérative rassemblant des activités et des productions variées; et c'était de la prolifération de ces institutions-modèles car rationnellement organisées dans un monde commercial chaotique, que devait venir le progrès social. Mais l'Etat (et Blanc avait en tête ici l'Etat démocratique représentant les intérêts de tous, dont l'institution du suffrage universel au printemps 1848 allait véritablement inaugurer l'existence) avait également plusieurs rôles majeurs à jouer dans cette évolution. Il était, en effet, le «régulateur suprême » de la production : il lui fallait, à l'origine fournir les dotations permettant la création des ateliers sociaux, il lui fallait aussi protéger leur première croissance en milieu de concurrence, notamment en commanditant l'association. Il lui revenait également de transmettre à l'association des principes de bon gouvernement, en s'attachant à instituer dans l'atelier les règles de la démocratie, représentation, élection, discussion. Il lui fallait encore pousser au respect maximal possible de l'égalité25, vérifier qu'en termes de garanties de travail et d'éducation les ouvriers, devenus collectivement propriétaires de l'association respectent, notamment à l'égard des nouveaux entrants, les principes d'une véritable liberté ; égalité et liberté étant les conditions du développement de comportements fraternels. Il fallait enfin à l'Etat instituer au sein des associations des règles permettant le développement d'une solidarité plus vaste, une solidarité concernant les associations entre-elles puis, au-delà, les industries entre-elles.

Le second volet du plan de réforme global des Socialistes Fraternitaires correspondait plus nettement au rôle que l'Etat devait jouer globalement dans l'économie. Dans l'Exposé, ils écrivaient, "Quant à l'Etat, il est clair que, s'il a une fonction sociale, c'est d'intervenir en protecteur pacifique partout où il y a des droits à équilibrer, des intérêts à garantir ; c'est de placer tous les citoyens dans des conditions égales de développement moral, intellectuel et physique. Voilà sa loi. Et il ne peut l'accomplir, cette loi, qu'en se réservant le droit de distribuer le crédit, de fournir des instruments de travail à ceux qui en manquent, de manière à rendre accessible à tous les sources vives de la richesse. Otez cette attribution économique, toute de prévoyance, à l'Etat, nous entendons l'Etat démocratiquement constitué, - et l'organisation du travail est un mensonge, et le remède aux maux intolérables du peuple est impuissant à toujours » (89). Le problème initial était de doter l'Etat des ressources susceptibles de l'aider à fonder les associations, sans recourir à l'impôt ou à l'emprunt; et le moyen était de lui transférer l'administration de pans entiers de l'économie nationale. La solution imaginée par Blanc, Vidal et Pecqueur conduisait toutefois à dépasser l'enjeu initial et à confier à l'Etat un rôle beaucoup plus important dans la conduite de l'économie. Dans ce second volet, on voyait l'Etat devenir progressivement l'institution première de la transformation sociale à opérer. Dans l'Exposé, les Socialistes Fraternitaires expliquaient que

\footnotetext{
${ }^{25}$ Blanc, mais aussi Pecqueur ou Vidal reviendront à plusieurs reprises en 1848 sur l'application possible dans le présent de leur principe égalitaire idéal de distribution. Ils conviendront que l'éducation présente des esprits rendait une complète application de ce principe très délicate; il fallait donc faire quelques concessions au principe concurrent considérant qu'il fallait un minimum de correspondance entre contribution et rétribution. Ils proposeront alors des solutions de compromis pour ces temps de transition et estimeront par exemple que (vérifier LCS).
} 
l'Etat devait bien sûr favoriser la multiplication des ateliers sociaux, mais il devait aussi racheter les usines en difficulté et «créer de nouveaux centres de travail et de production » pour toutes les populations industrieuses en difficulté. Le moyen adopté consistait à racheter les chemins de fer, les mines et les canaux. L'Etat devait fonder et diriger des colonies agricoles sur tout le territoire. Le projet prévoyait également « de transformer le système des banques et des assurances en institutions nationales » (92) et à placer l'intégralité des échanges et du commerce sous le contrôle de l'Etat par la constitution d'entrepôts et de bazars. Toutes ces transformations permettaient de doter l'Etat d'un budget assurant le financement des associations. Mais elles conduisaient aussi à faire de cet Etat le principal acteur de l'économie, un financeur, mais également un producteur. Ce second volet du plan de réforme institutionnelle correspondait plus exactement aux projections imaginées par Pecqueur, notamment dans son grand ouvrage, Théorie nouvelle d'économie sociale (1842). Et, sur ce chapitre Vidal semble ici très proche de Pecqueur : les neufs grands points du plan général de réforme sociale qu'il présentera dans Vivre en travaillant à la suite de l'expérience du Luxembourg signalent surtout cette croissance de l'Etat dans l'économie.

Ces transformations institutionnelles permettaient la réalisation graduelle des principes de justice. Elles avaient conduit à définir les deux institutions piliers, l'Association et l'Etat, à proposer une division des tâches entre ces deux institutions, et donc à définir une logique du développement normal des sociétés industrielles et démocratiques. Toutefois, comme l'ont signalé les passages précédents, l'Exposé était un texte de synthèse et il dissimulait certaines tensions théoriques et doctrinales qui pouvaient exister entre Blanc, Pecqueur et Vidal. Pour Blanc, le grand enseignement du Luxembourg avait été la réalisation à grande échelle d'expériences coopératives. Cela venait confirmer l'idée qu'on pouvait «se servir de l'arme même de la concurrence, pour faire disparaître la concurrence », la mise en concurrence des ateliers sociaux et des compagnies privées ne pouvant conduire qu'à la victoire des premiers et à leur prolifération. Blanc retrouvait dans cette idée de micro-expériences exemplaires destinées à proliférer un thème qui était commun à cette génération de penseurs socialistes, des fouriéristes à Philippe Buchez ou François-Vincent Raspail ; mais Blanc insistait aussi sur la nécessaire métamorphose des fonctions économiques de l'Etat pour accompagner ce processus. Dans ce processus, l'Etat démocratique, tel qu'il se dessinait en 1848, était principalement l'instigateur et le protecteur des premières victoires des associations. Une division des fonctions se manifestait donc entre la société civile et l'Etat et la vision de Blanc ne semblait pas inclure comme horizon de l'évolution la fusion des associations dans l'Etat. Le point de vue que proposait Pecqueur était sensiblement différent. Il relevait le rôle initial des associations, "le mouvement par en bas", mais signalait que le processus conduisait rapidement à une intégration, un «mouvement par en haut» ${ }^{26}$. Et dès 1842 , il traçait les lignes d'un programme qui, bien qu'intégrant comme celui de Blanc les idées d'association et d'Etat démocratique, n'en était pas moins différent: "Socialiser et nationaliser tous les instruments de travail, ramener l'activité et la production de tous les citoyens sans exception à l'unité et à la centralisation gouvernementale: il suffit de ces deux grandes mesures pour obtenir la solution facile de tous les problèmes jusqu'ici insolubles de l'économie politique et sociale : l'équilibre constant de la production et de la consommation; l'accroissement ou le mouvement toujours proportionnel de la population et des moyens d'existence; l'égalité naturelle et obligée des conditions ; l'hérédité des fonctions selon l'esprit ou le mérite » (1842: 675-676). Chez Pecqueur, bien qu'une nouvelle fois des nuances puissent être apportées, le

\footnotetext{
26 «D’abord viendra le mouvement spontané, multiple, libre et fortuit de chacun et de tous, dans le sens providentiel de l'association par groupes ou corporations ; puis le souverain, l'Etat-Peuple, se manifestant dans son unité collective, ramassera et condensera, en quelque sorte, par l'action centripète de sa force unifiante, tous ses éléments épars, tout ce travail incohérent, où se forme l'embryon organique » SP.
} 
terme de l'évolution sociale résidait donc plutôt dans la fusion graduelle de la société civile dans l'Etat collectiviste ${ }^{27}$.

\section{Why not Socialism?}

Les socialistes français antérieurs à 1851 sont sortis des radars de l'histoire de la pensée économique après 1945. Dans son History of Economic Analysis, Joseph Schumpeter notait à propos de ces socialistes, « some of the utopists ideas were in fact unalloyed non-sense - in several cases definitely pathological non-senses - and hardly any of them can be taken quite seriously» ${ }^{28}$; dans ce même ouvrage, Schumpeter congédiait la traditionnelle histoire des doctrines économiques qui, avec Charles Gide et Charles Rist, par exemple, avait longtemps accordé un grande place à ces socialistes du premier 19e siècle. L'accent porté par Schumpeter sur l'analyse (une boite à outil indépendante de toutes options politique, morale, religieuse...) conduisait à se focaliser sur la dimensions « mécanique » de l'économie, et, dans le contexte des années 50, sur la théorie de l'équilibre général. Mais le récent rééquilibrage de la discipline économique proposée par Amartya Sen qui invitait à balancer la dimension «mécanique » (« engineering approach») et la dimension «éthique » ré-ouvre une voie d'accès à ces auteurs socialistes du premier $19^{\mathrm{e}}$ siècle ${ }^{29}$. En effet, pour l'historien de la pensée économique, une façon d'accéder aujourd'hui à ces œuvres est de considérer qu'elles firent avancer la réflexion sur la question des principes et des institutions d'une société juste.

Peut-on aller plus loin et jeter quelques passerelles avec les débats modernes? Oui, mais avec prudence et, dans le cadre de cette conclusion, seules quelques pistes et préliminaires peuvent être mentionnés. Louis Blanc faisait de Rousseau le «précurseur du socialisme moderne $»^{30}$, et Pecqueur revendiquait également l'exemplarité du modèle du « contrat social ». Ces références peuvent conduire à risquer quelques rapprochements entre les thèses des Fraternitaires et les théories modernes libérale-égalitaire qui, à la suite du travail pionnier de John Rawls ${ }^{31}$ conduisirent à une nouvelle formulation des principes et des institutions d'une société juste ${ }^{32}$. Au sein de ces débats modernes, plusieurs rapprochements pourraient être tentés, et, par exemple, l'emploi de la notion de pouvoir par les Fraternitaires mériterait une comparaison un peu systématique avec les notions de capabilité ou de fonctionnement qui permettent à un auteur comme Amartya Sen de discuter l'idée de biens premiers chez Rawls. Toutefois un rapprochement plus évident encore s'impose avec les thèses de Gerald Gohen. L'une des proximités les plus intéressantes concerne la discussion sur le principe de différence et l'idée défendue par Cohen que la correspondance entre capacités et rétributions n'a rien d'automatique, bien au contraire, dans une société qui se prétend

\footnotetext{
${ }_{27}$ Voir par exemple les analyses présentées dans Le Salut du peuple. Mais en 1842, il notait déjà, «le propre de l'association, c'est le concert obligé, l'harmonie, la hiérarchie, l'unité dans le but, dans les moyens et dans les efforts de l'activité collective. Mais là où est tout cela, là se trouve aussi nécessairement l'autorité, le centre, une force qui dirige, qui attire et qui renvoie » (TN, p. 457).

${ }_{28}$ Mais Schumpeter ajoutait, "though an exception should perhaps be made in favor od L. Blanc », J. Schumpeter, History of Economic Analysis, [1954], Taylor and Françis, 2006, p. 430.

${ }^{29}$ Amartya Sen, On Ethics and Economics, Oxford, Blackwell, 1987.

${ }^{30}$ Louis Blanc, Histoire de la révolution française, Paris, Langlois et Leclercq, 1847, vol. 1, p. 399.

${ }^{31}$ Rawls qui, on le sait, dans les premières pages de son ouvrage pionnier écrivait, "what I have attempted to do is to generalize and carry to a higher order of abstraction the traditional theory of social contract », $\boldsymbol{A}$ Theory of Justice, Oxford, Oxford University Press (revised edition), 1999, p. XVIII.

${ }^{32}$ Pour un tableau éclairant, Philippe Van Parijs, «Qu'est ce qu'une société juste ? La pensée philosophique contemporaine », Conférence aux semaines sociales de France, 2006 ; également, Christian Ansperger et Philippe Van Parijs, Ethique économique et sociale, Paris, La Découverte, 2000. Noter également l'attention aux conséquences chez les Fraternitaire et proximité ici avec Louvain.
} 
évoluée ${ }^{33}$. Dans Why not Socialism?, Cohen défend le principe de "l'égalité socialiste des chances $»^{34}$ et complète même ce principe (qui permet encore certaines inégalités) en lui ajoutant la nécessité d'un principe communautaire fort conduisant (en contrôlant les quelques inégalités permises encore par le principe de l'ESG) à développer et renforcer les sentiments de réciprocité, de mutualité et de solidarité. Cohen, qui souligne que le marché et la concurrence reposent sur prédation et cupidité, signale donc le caractère souhaitable du socialisme mais s'interroge plus nettement sur son caractère faisable, signalant les problèmes posés par l'état de la technologie sociale. Sur ce point le détour par les Fraternitaires permettrait sans doute d'en partie ruminer ce relatif pessimisme car, l'une des particularité de ces auteurs, et de tous ces socialistes antérieurs à 1851, a été de développer une imagination sociale (pas toujours folle ou farfelue) pour penser changement et transition dans un monde en crise. Mais le rapprochement avec Cohen peut également s'autoriser d'une constatation plus circonstancielle: on trouve chez Louis Blanc une "expérience de pensée » similaire au fameux modèle du camping qui inaugure la réflexion de Cohen dans son Why not Socialism? Dans son Histoire de la Révolution de 1848, Blanc raconte en effet l'anecdote suivante : engagé, un jour, dans un débat avec l'un de ses amis Anglais lui faisant part de son extrême scepticisme, pour dire le moins, sur les idéaux généreux du socialisme, notamment en matière de comportement humain, ils se retrouvaient tous deux, le lendemain, pour un piquenique dont le déroulement permis au final à Louis Blanc de faire douter son contradicteur Anglais :

«Il arriva que, le lendemain, devait avoir lieu une partie de campagne dont nous étions, mon interlocuteur et moi. Il s'agissait d'aller diner sur l'herbe dans l'ile où fut signée la grande charte des libertés anglaises, magna Charta. Notre caravane se composait de personnes des deux sexes, fort différent par la position sociale, la fortune, l'intelligence, mais que rapprochaient un sentiment de bienveillance mutuelle et éclairée. Il y avait un chiffre de dépense à atteindre, des provisions à rassembler : ce fut l'affaire de qui avait du vin dans ses caves et de l'argent dans ses bourses. A l'heure dite, nous étions tous au rendez-vous convenu. Des barques nous attendaient; nous y montâmes, nous partîmes. Ceux qui savaient ramer se mirent aux rames sans se faire prier, et, tandis qu'ils se fatiguaient joyeusement à conduire, les autres s'occupaient à regarder fuir les bord de la Tamise, à jouer avec les enfants ou à causer avec les dames.

Quand nous eûmes mis pied à terre et qu'il fut question de transporter à l'endroit désigné d'avance nos munitions... de paix, les plus vigoureux réclamèrent le privilège de se charger du précieux fardeau, chacun comptant bien, d'ailleurs, avoir son tour dans le bonheur d'être utile. C'est ce qui arriva. Aux dames échut de droit le soin de tout disposer pour notre festin champêtre, et les enfants trouvèrent parfaitement à s'employer, à leur grande joie. Il va sans dire que, pendant le repas, la bonne harmonie ne fut troublée par aucune prétention tirée, soit du plus ou moins de mérite de tel convive, soit de la différence des petits services rendus ou à rendre. Les rameurs, qui s'étaient donnés tant de peine, n'auraient eu garde d'en prendre texte pour réclamer quoi que ce fût au détriment de ceux qui n'avaient point ramé. Les plus belles attirèrent davantage les regards, peut-être ; mais il eût paru fort étrange, qu'en raison de leur beauté elles exigeassent les meilleurs vins et les mets les plus délicats. Chacun mangea selon son appétit, but selon sa soif. Il fallut pourvoir ensuite à ce que la soirée fût agréablement

\footnotetext{
${ }^{33}$ Gerald Cohen, If You're an Egalitarian, How Come You're So Rich ?, Havard University Press, 2001.

${ }^{34}$ Cette égalité socialiste des chances (plus large que «l'égalité bourgeoise des chances» et que "l'égalité de gauche libérale »), «vise à corriger tous les désavantages subis, tous ceux dont le sujet ne saurait être tenu pour responsable, qu'il s'agisse de handicaps sociaux ou naturels »(23). Les seules inégalités demeurent donc les conséquences des choix et arbitrages individuels concernant l'alternative travail/loisir.
} 
remplie, et chacun y fit de son mieux. L'un chanta, l'autre joua de la guitare, un troisième récita des vers charmants de sa façon. Personne ne fut requis de faire ce qu'il ne savait pas, personne ne refusa de faire ce qu'il savait, et tous reçurent de cordiales félicitations, quoique tous n'eussent contribué aux plaisirs de la journée, ni au même degré, ni de la même manière. Bref, nous nous en revînmes enchantés d'avoir passé ensemble des heures si douces et nous promettant de recommencer le plus tôt possible $»^{35}$.

${ }^{35}$ Louis Blanc, Histoire de la révolution de 1848, ouv. cit., p. 155-156. 